Patients with left heart disease commonly develop pulmonary hypertension (PH), and some subsequently develop pre-capillary vascular remodelling. This combined pre and post capillary pulmonary hypertension (Cpc- $\mathrm{PH})$ is defined as mean pulmonary artery pressure (mPAP) $\geq 25 \mathrm{mmHg}$, pulmonary arterial wedge pressure (PAWP) $>15 \mathrm{mmHg}$ and diastolic pulmonary gradient (DPG) of $\geq 7$. Patients with $\mathrm{Cpc}-\mathrm{PH}$, have a worse outcome and targeted pulmonary vascular therapies may be useful. The aim of this study was to assess MRI measured septal angle in the assessment of $\mathrm{Cpc-PH}$.

Methods Consecutive, incident suspected $\mathrm{PH}$ patients who underwent MRI at a pulmonary hypertension referral centre from April 2012 to October 2015 were assessed. Patients with PAWP $>15 \mathrm{mmHg}$, with right heart catheter and MRI on the same day were included. The diagnostic accuracy of septal angle to identify $\mathrm{Cpc}-\mathrm{PH}$ was assessed.

Results 2437 patients underwent MRI, 1272 were incident and 227 patients had PAWP >15 mmHg. 163 had MRI and right heart catheter on the same date. The average age was 70 (sd 11), 64\% were female. Systolic interventricular septal angle correlated with DPG $(r=0.735, \mathrm{p}<0.0001)$. ROC analysis showed septal angle was predictive of $\mathrm{Cpc}-\mathrm{PH}$ (defined by DPG $\geq 7)$ with area under the curve $0.90(p<0.0001)$. Analysis of the ROC data showed $160^{\circ}$ septal angle as a threshold predicted a DPG of $\geq 7 \mathrm{mmHg}$, with $74 \%$ sensitivity and $90 \%$ specificity $(\mathrm{p}$-value $<0.0001)$. Systolic interventricular septal angle was predictive of outcome with univariate hazard ratio 1.017 (95\% CI 1.007-1.028, $\mathrm{p}=0.001)$. Dichotomised by median value $\left(149^{\circ}\right)$ the hazard ratio was 3.245 (95\% CI 1.720-6.119, p<0.0001) and log-rank chi-square was 12.387 . Conclusion Interventricular septal angle on MRI can non-invasively predict the presence of an elevated diastolic pressure gradient in patients with left heart disease.

\section{S49 ELICITATION OF DISUTILITY VALUES ASSOCIATED WITH THE MODE OF ADMINISTRATION OF DRUGS ACTING ON THE PROSTACYCLIN PATHWAY IN PULMONARY ARTERIAL HYPERTENSION (PAH)}

${ }^{1}$ EW Davies, ${ }^{2} \mathrm{~S}$ Llewellyn, ${ }^{1} \mathrm{~A}$ Beaudet, ${ }^{2} \mathrm{CE}$ Kosmas, ${ }^{2} \mathrm{H}$ Doll. ${ }^{7}$ Actelion Pharmaceuticals Ltd., Allschwil, Switzerland; ${ }^{2}$ ICON plc, Abingdon, UK

\subsection{6/thoraxjnl-2017-210983.55}

Introduction and Objectives Pulmonary arterial hypertension $(\mathrm{PAH})$ is a rare, incurable disease associated with decreased life expectancy and a marked impact on quality of life (QoL). There are three classes of drugs available for treatment: endothelin receptor antagonists (ERA), drugs acting on the nitric oxide pathway (riociguat and phosphodiesterase type 5 inhibitors (PDE5i)), and drugs acting on the prostacyclin pathway. The latter have widely different modes of administration continuous intravenous infusion, continuous subcutaneous infusion, inhaled and oral - which could be associated with varying treatment burden. Health economic evaluations should incorporate the QoL impact of how drugs are administered. This study aimed to elicit societal utility values associated with different routes of administration of drugs acting on the prostacyclin pathway for use in economic evaluations of $\mathrm{PAH}$ treatments.

Methods A UK general public sample completed the EQ-5D$5 \mathrm{~L}$ and valued four health states in Time-Trade Off interviews. The health states (drafted from literature and interviews with
PAH experts $(n=3)$ contained identical descriptions of PAH and ERA/PDE5i treatment, but differed in description of prostacyclin treatment administration including: oral (tablets), inhaled (nebulizer), continuous subcutaneous infusion, and continuous intravenous infusion.

Results A total of 150 participants (63\% female; mean age 37 years) completed interviews. Utilities are presented as values between 1 and 0 , where 1 is equal being in a state of 'full health' and 0 is equal to being dead. The mean (SD) utility for the oral health state was $0.84(0.16)$, while the other health states were all significantly lower at $0.73(0.27)$ for inhaled $(p=0.001), 0.58(0.31)$ for subcutaneous $(p<0.001)$, and $0.54(0.32)$ for intravenous $(\mathrm{p}<0.001)$. Utility differences compared to the oral health state showed that there are disutilities (negative differences) associated with the inhaled, subcutaneous, and intravenous continuous modes of treatment administration. Disutilities were -0.11 for inhaled, -0.26 for subcutaneous, and -0.30 for intravenous administration.

Conclusion The Results demonstrate quantifiable QoL differences between modes of administration of drugs acting on the prostacyclin pathway, so as to allow appropriate reflection of the unique QoL burden within an economic evaluation of drugs for PAH treatment.

\section{S50 CAMPHOR SCORE: SUSTAINED IMPROVEMENT IN PATIENT REPORTED OUTCOMES FOLLOWING PULMONARY ENDARTERECTOMY IN CHRONIC THROMBOEMBOLIC PULMONARY HYPERTENSION}

${ }^{1} \mathrm{M}$ Newnham, ${ }^{1} \mathrm{~K}$ Bunclark, ${ }^{1} \mathrm{~N}$ Abraham, ' $\mathrm{L}$ Amaral Almeida, ${ }^{1} \mathrm{~J}$ Cannon, ${ }^{1} \mathrm{~S}$ Clare, ${ }^{1} \mathrm{~N}$ Doughty, ${ }^{1} \mathrm{~J}$ Dunning, ${ }^{1} \mathrm{C} \mathrm{Ng},{ }^{1} \mathrm{~A}$ Ponnaberanam, ${ }^{2} \mathrm{~S}$ Scholtes, ${ }^{1} \mathrm{~K}$ Sheares, ${ }^{1} \mathrm{~N}$ Speed, 'D Taboada, 'M Toshner, 'S Tsui, 'D Jenkins, 'J Pepke-Zaba. 'Papworth Hospital, Cambridge, UK; ${ }^{2}$ University of Cambridge, Cambridge, UK

\subsection{6/thoraxjnl-2017-210983.56}

Introduction The CAMPHOR (Cambridge Pulmonary Hypertension Outcome Review) score is an internationally validated patient reported outcome (PRO) measure for pulmonary hypertension, including chronic thromboembolic pulmonary hypertension (CTEPH). It assesses 3 areas: activity (score 0 30 ), symptoms (0-25) and quality of life (QoL) (0-25); with a higher score indicating a worse PRO. CTEPH frequently causes debilitating symptoms and functional impairment, which can be improved in selected patients with pulmonary endarterectomy (PEA). However, a subset will have residual pulmonary hypertension. We aim to assess PROs in patients with CTEPH undergoing PEA.

Methods Consecutive CTEPH patients undergoing PEA from June 2006 to August 2016 at the UK National PEA centre, were included in this retrospective analysis. Patients are reviewed after PEA every 6-12 months for at least 5 years. CAMPHOR scores were recorded prospectively when patients attended hospital assessment and at each follow-up, ensuring high capture.

Results 1151 patients underwent PEA during the study period. Of those, $937(81 \%)$ had a CAMPHOR score recorded at baseline (pre-PEA) and 816 (77\% of 1059 alive) at follow-up within a year of PEA (post-PEA). We confirmed significant improvements in $6 \mathrm{~min}$ walk distance and haemodynamics post-PEA (pre/post-PEA median \pm IQR: 6 mwd 300 199 Metres/360 $\pm 165 ; \quad$ mPAP $45 \pm 15 / 25 \pm 13 \mathrm{mmHg} ; \quad$ PVR 669 $\pm 478 / 246 \pm 214$ dynes.s.cm ${ }^{-5} ;$ CI $2.2 \pm 0.8 / 2.3 \pm 0.7 \mathrm{~L} / \mathrm{min} / \mathrm{m}^{2}$ ). The difference in median CAMPHOR scores pre- and post- 